\title{
Papillary Carcinoma of the Breast: An Overview
}

\author{
Sumanta Kumar Pal, M.D., Sean K. Lau, M.D., Laura Kruper, M.D., Uzoamaka Nwoye, M.D., \\ Carlos Garberoglio, M.D., F.A.C.S., Ravi K. Gupta, B.Sc., Benjamin Paz, M.D., F.A.C.S., Lalit \\ Vora, M.D., Eduardo Guzman, M.D., Avo Artinyan, M.D., M.Sc., and George Somlo, M.D., \\ F.A.C.P.
}

\begin{abstract}
Papillary carcinoma of the breast represents approximately $0.5 \%$ of all newly diagnosed cases of breast cancer. The prevalence of both invasive and in situ papillary carcinoma seems to be greater older postmenopausal women, and -in relative terms-in males. Histologic features of the tumor include cellular proliferations surrounding fibrovascular cores, with or without invasion. In this review, characteristics of both in situ and invasive disease are outlined. Immunohistochemical analyses of papillary carcinoma suggest the utility of markers such as smooth muscle myosin heavy chain, calponin, p63 and high molecular weight keratins, which can characterize the myoepithelial cell layer. With respect to radiographic evaluation of papillary carcinoma, ultrasonography is the most extensively studied imaging modality, though magnetic resonance mammography has potential utility. Available data suggest improved outcome for papillary carcinoma as compared to invasive ductal carcinoma. Treatment-related information for patients with papillary carcinoma is limited, and patterns noted in available series suggest a variable approach to this disease. The scarcity of information underscores the need for further treatmentand outcome-related studies in papillary carcinoma of the breast.
\end{abstract}

\section{Keywords}

papillary; breast carcinoma; male breast cancer; breast ultrasonography; breast magnetic resonance mammography

\section{INTRODUCTION}

In 2009, an estimated 194,280 cases of invasive breast cancer were diagnosed.[1] Included in this estimate is a heterogeneous array of pathologic diagnoses, each with a distinct prognosis and management strategy. Papillary breast cancer represents approximately $0.5 \%$ of these invasive breast cancers, typically presenting with bloody nipple discharge, an abnormal mass, or radiographic abnormalities.[2] Histological characterization suggests proliferations of cells arranged around fibrovascular cores, grossly forming a circumscribed mass.[3] Distinction of invasive papillary carcinoma from non-invasive forms is critical, as each entity carries a unique prognosis. However, large series often include an amalgam of these diagnoses, given their relatively low cumulative frequency.[2, 4] A separate challenge in characterizing papillary carcinoma is related to the fact that a large proportion of the literature devoted to this entity is comprised of individual case reports. Herein, we provide a

Corresponding Authors: Sean K. Lau, M.D., Staff Pathologist, City of Hope Comprehensive Cancer Center, 1500 East Duarte Road, Duarte, CA 91010, Office: (626) 256-4673, slau@ @ coh.org. George Somlo, M.D., F.A.C.P., Professor and Director, Breast Oncology, Department of Medical Oncology \& Therapeutics Research, Co-Director, Breast Cancer Program, City of Hope Comprehensive Cancer Center, 1500 East Duarte Road, Duarte, CA 91010, Office: (626) 256-4673, gsomlo@ coh.org.

Disclaimers: None 
comprehensive summary of data related to relevant subtypes of papillary carcinoma, with a discussion of diagnosis, prognosis and management.

\section{DIAGNOSIS}

\section{Pathologic Features}

In the context of breast pathology, the term papillary carcinoma encompasses a morphologically heterogeneous group of lesions, all of which share a growth pattern characterized by the presence of arborescent fibrovascular stalks lined by epithelial cells. Malignant papillary neoplasms of the breast comprise a number of microscopically distinct lesions including ductal carcinoma in situ (DCIS) arising in an intraductal papilloma, papillary DCIS, enscapsulated papillary carcinoma, solid papillary carcinoma, and invasive papillary carcinoma.[5-7] All malignant papillary proliferations of the breast lack an intact myoepithelial cell layer within the papillae, an important feature which allows distinction from benign intraductal papillomas.

Otherwise benign-appearing intraductal papillomas may display proliferative areas which would satisfy criteria for DCIS if observed outside of the context of a papillary lesion. These areas of DCIS are generally composed of uniform appearing cells with low or intermediate grade nuclear aytpia, typically with a solid or cribriform growth pattern. At present, there are no universally accepted guidelines for diagnosing a papilloma with DCIS. Proposed criteria have included the presence of DCIS greater than $3 \mathrm{~mm}$ in size,[8] and DCIS comprising at least a third but less than $90 \%$ of the papillary lesion.[7] Papillary lesions exhibiting atypical features not meeting these thresholds have been classified as atypical papillomas. In contrast, others advocate rendering a diagnosis of DCIS arising in a papilloma regardless of the size or extent of the involved area.[6]

Papillary DCIS is characterized by the presence of fibrovascular fronds lined by neoplastic epithelium (Figure 1). Features of an underlying pre-existing benign papilloma are not present. The lining epithelium is typically comprised of monomorphic, stratified columnar cells; however, solid, cribriform, or micropapillary proliferations may also be observed. Nuclei are usually of low or intermediate grade. The papillae are devoid of myoepithelial cells, though as with other morphologic types of DCIS, a myoepithelial layer is retained at the periphery of the involved duct. Lesions are frequently multifocal and peripheral in distribution.

Encapsulated papillary carcinoma, also known as intracystic papillary carcinoma, is the term used to describe a solitary, centrally located malignant papillary proliferation involving a cystically dilated duct. Histologically, the lesion is well circumscribed, with the involved duct surrounded by a thick fibrous capsule (Figure 2). The duct is filled by slender fibrovascular stalks lacking myoepithelial cells. Various patterns of epithelial proliferation may be observed, including stratified spindle cell, cribriform and solid arrangements. Low or intermediate nuclear grade is typical of these lesions, with high grade nuclear atypical rarely observed. Although morphologically well delineated and traditionally considered to represent a variant of DCIS, immunohistochemical studies have failed to consistently demonstrate the presence of a myoepithelial cell layer at the periphery of encapsulated papillary carcinomas. The absence of myoepithelial cells has led some investigators to propose that many encapsulated papillary carcinomas are not in situ lesions, but rather invasive carcinomas with a circumscribed nodular histology. $[9,10]$ Other authors, however, feel encapsulated papillary carcinomas are best considered in situ carcinomas despite the absence of surrounding myoepithelial cells based on the finding of an intact basement membrane, as shown by collagen type IV expression, at the periphery of the majority of 
encapsulated papillary carcinomas, as well as the demonstrated clinically indolent behavior of these lesions.[7, 11]

A minority of encapsulated papillary carcinomas may be associated with a component of invasive carcinoma (invasive carcinoma arising in an encapsulated papillary carcinoma). The invasive component is characterized by an infiltrative appearance with extension beyond the fibrous capsule of the lesion and an associated stromal reaction (Figure 3). Invasive areas in general do not display papillary features, but rather exhibit the morphology of an invasive ductal carcinoma, not otherwise specified. In cases of encapsulated papillary carcinomas with associated invasion, it is currently recommended that staging be determined based on the sized of the invasive component only, without consideration of the encapsulated component of the tumor, in order to prevent overtreatment.[5, 6] In such circumstances, to avoid confusion and ensure appropriate clinical management, one may prefer to report only the size of the unequivocal invasive focus of carcinoma when rendering a final diagnosis; for example, "invasive ductal carcinoma, not otherwise specified $(x \mathrm{~cm})$, arising in association with an encapsulated papillary carcinoma."

Solid papillary carcinoma appears microscopically as well circumscribed, densely cellular, expansile nodules of epithelial cells (Figure 4).[12,13] The neoplastic cells are oval or spindle shaped, exhibit low to intermediate grade nuclear atypia, and have a monotonous appearance (Figure 5). Many cases exhibit neuroendocrine features characterized by argyrophilia and immunoreactivity for chromogranin A. Associated intracellular and extracellular mucin is also a common finding. Although no discrete papillary structures are present, an underlying fibrovascular stromal network is typically observed, supporting classification of the lesion as papillary, despite its solid morphologic appearance. Interestingly, while the nodular appearance of solid papillary carcinoma was initially thought to result from proliferating neoplastic cells involving large or dilated ducts, immunohistochemical studies have demonstrated an absence of myoepithelial cells at the periphery of the nodules in some cases.[13-15] As with encapsulated papillary carcinoma, the apparent absence of myoepithelial cells in a subset of solid papillary carcinomas has prompted the suggestion that solid papillary carcinomas may represent invasive tumors with pushing borders, rather than purely in situ intraductal lesions. Solid papillary carcinomas are often accompanied by associated areas of invasive carcinoma. The invasive component most frequently manifests as a mucinous or neuroendocrine like carcinoma, though other histologic types of invasive carcinoma may also be observed.[12, 13]

The term invasive papillary carcinoma is reserved for infiltrating breast carcinomas exhibiting an exclusively papillary morphology, and should be distinguished from the other malignant papillary lesions described previously. Defined as such, invasive papillary carcinomas are extremely rare.[16] Encapsulated and solid papillary carcinomas are not currently classified as invasive papillary carcinomas, though as previously discussed, a subset of these tumors may represent low grade carcinomas exhibiting an expansile type of invasion. Invasive papillary carcinoma should not be confused with invasive micropapillary carcinoma, which is a clinically and pathologically separate entity. In contrast with invasive papillary carcinoma, invasive micropapillary carcinoma morphologically lacks true fibrovascular cores, and is characterized by neoplastic cells arranged in solid nests or tubules surrounded by clear spaces (Figure 6). The distinction of invasive papillary from micropapillary carcinoma has relevant clinical implications as the latter is considered an aggressive form of mammary carcinoma frequently associated with lymph-vascular invasion and axillary lymph node metastases.[17-19]

Pathologic characterization of papillary lesions of the breast is based primarily on morphologic considerations. In particular, loss of myoepithelial cells within the 
fibrovascular papillae is the most important feature for the identification of malignant papillary proliferations and their separation from benign intraductal papillomas.

Myoepithelial cells are, however, frequently difficult to discern on routine hematoxylin and eosin stained preparations. As such, immunohistochemistry is often utilized as an adjunct for evaluating the presence and distribution of myoepithelial cells in papillary neoplasms of the breast. Papillomas exhibit distinct, uniform staining of myoepithelial cells within the constituent papillae of the lesion as well as surrounding the periphery of the involved duct in a contiguous fashion (Figure 7). In contrast, malignant papillary proliferations generally lack immunohistochemical expression of myoepithelial cell associated antigens within the papillary processes, though focal or patchy areas of immunoreactivity may be present in cases of DCIS arising within a preexisting benign intraductal papilloma. Myoepithelial cells are not consistently detected at the peripheral aspects of papillary DCIS, encapsulated papillary carcinoma, and solid papillary carcinoma, and partial, discontinuous, or absent staining may be observed in these particular lesions (Figure 8). There are a number of markers that identify myoepithelial cells, the most useful of which include calponin, smooth muscle myosin heavy chain, and p63.[5, 13, 20] The individual markers exhibit variable sensitivity and also show different degrees of cross reactivity with cell types other than myoepithelial cells such as stromal myofibroblasts, pericytes and vascular smooth muscle cells, which can potentially complicate interpretation of positive staining. Most laboratories thus employ a panel of several myoepithelial cell markers when evaluating difficult papillary lesions.

Antibodies directed against high molecular weight keratins, including keratin 5/6, keratin 14 , and $34 \beta E 12$ have also been utilized in the immunohistochemical assessment of papillary lesions of the breast. These markers are particularly useful in the context of distinguishing usual ductal hyperplasia in a benign intraductal papilloma from papilloma with DCIS and solid papillary carcinoma. Areas of usual ductal hyperplasia in a papilloma are typically characterized by strong staining of the majority of the epithelial cells in a mosaic pattern, while in contrast, malignant papillary proliferations demonstrate diminished to absent expression of high molecular weight keratins by immunohistochemical methods.[14, 21-23] It has recently been suggested that evaluation of estrogen receptor (ER) expression may be a useful adjunct to high molecular weight keratin staining, as well. In one study, a series of 82 core biopsies from papillary lesions were subjected to immunohistochemical analysis.[24] In this series, a combination of ER-low/keratin 5-high staining characterized nonatypical papillomas, while a pattern of ER-high/keratin 5-low staining was more frequently observed in ayptical papillary lesions. In a validation cohort including 30 papillary specimens, 29 cases were correctly classified using this scheme.

\section{Genetic Characteristics}

Genetic features may also aid in distinguishing papillary carcinoma from benign lesions. An assessment of four microsatellite markers in 15 specimens (representing a spectrum of papillary lesions) suggested that loss of heterozygosity ( $\mathrm{LOH}$ ) of 16q23 was specific to malignant lesions.[25] $\mathrm{LOH}$ at the TP5 locus was also found to be significantly associated with a malignant phenotype $(\mathrm{P}=0.01)$. A separate analysis of 10 benign papillomas and 14 papillary carcinomas assessed chromosomes $3,7,17$ and $\mathrm{X}$ using multicolor fluorescent in situ hybridization.[26] Alterations in all four chromosomes were present in all cases of DNA-aneuploid carcinoma. Thus, DNA-ploidy, in association with changes at specific loci, may be informative in the diagnosis of papillary carcinoma.

\section{Radiographic Findings}

Several reports characterize papillary carcinoma using a variety of modalities. With respect to ultrasound, a report of 42 cases of papillary neoplasms suggests three basic 
ultrasonographic profiles: (1) intraductal mass with or without ductal dilatation, (2) intracysic mass, and (3) solid pattern with an intraductal mass completely filling the duct. [27] Benign papillomas were noted to have denser and coarser calcifications. In contrast, papillary carcinomas (found primarily in older age groups) were noted to have a larger solid component and a higher frequency of spontaneous intracystic bleed (Figure 9a). A larger experience reported ultrasonographic data from 46 patients with benign papillomas and 22 patients with papillary carcinomas.[28] In both groups, the shape of lesion was predominantly round or oval, and the margin appeared circumscribed. However, a nonparallel orientation, an echogenic halo, posterior acoustic enhancement and associated microcalcification were more frequently found in malignant as compared to benign lesions $(\mathrm{P}<0.05)$. When these features were used to define malignancy, a sensitivity and specificity of $85.7 \%$ and $64.9 \%$ was determined, respectively. Comparison of ultrasonography to traditional mammography appears to suggest enhanced sensitivity of the former modality. An assessment of 56 specimens excised from 40 patients with papillary tumors suggested detection of $82.1 \%$ of these lesions by ultrasound as compared to $37.5 \%$ by mammography. [29]

More limited experience is available for magnetic resonance classification of papillary tumors. A study of magnetic resonance mammography data from 468 patients included 27 $(5.7 \%)$ cases of rare breast neoplasms, with $5(1.0 \%)$ cases of invasive papillary neoplasms. [30] In general, distinguishing morphologic and kinetic features of rare breast neoplasms were infrequently elicited on magnetic resonance mammography. Rare breast lesions were largely characterized as benign. Larger analyses of magnetic resonance mammography in rare histologic subtypes, including papillary carcinoma, may clarify the utility of this modality in this setting. Figure $9 \mathrm{~b}$ illustrates a representative case of magnetic resonance mammography in a patient with invasive papillary carcinoma.

\section{Core Biopsy versus Surgical Excision}

There is some controversy regarding the optimal management of papillary lesions diagnosed by core-needle biopsy, with some authors advocating observation for benign disease and others suggesting surgical excision of all lesions in the same setting.[31] A study conducted at St. Vincent's Comprehensive Cancer Center examined 71 papillary lesions identified by core needle biopsy that were later surgically excised. Of these lesions, 47 were benign at the time of core biopsy. At the time of surgical excision, however, 4 cases (9\%) revealed malignancy and 13 cases (28\%) demonstrated atypical features. Of 13 lesions characterized as atypical at the time of core biopsy, surgical excision revealed malignancy in 7 lesions (54\%). Overall, it was noted that $38 \%$ of cases were up-graded between assessment at core needle biopsy and at the time of surgical excision. Thus for these lesions, surgical excision should be considered, especially if they are clinically or histologically worrisome.

\section{EPIDEMIOLOGY}

\section{Registry Analyses}

Data from the Netherlands Cancer Registry offer some perspective on demographics, stage distribution and outcomes for patients with papillary carcinoma.[2] From this registry, a total of 1,078 cases of papillary carcinoma were identified. Notably, the search term papillary carcinoma included six ICD-O codes, corresponding to papillary carcinoma NOS (8050), papillary adenocarcinoma (8260), papillary cystadenoma (8450), intraductal papilloma (8503), intracystic papillary adenoma (8504) and intraductal micropapillary carcinoma (8507). With this heterogeneous grouping, 159 patients were below the age of 50 (15\%), 356 patients were between 50-69 (33\%), and 563 patients were age 70 or greater $(52 \%)$. The majority of patients were diagnosed with early stage disease, with 488 patients 
classified as stage I (45\%) and 478 patients classified as stage II (44\%). Only 27 patients (3\%) were classified as having metastatic disease. Tumor grade was unknown in the majority of patients (59\%); in those cases in which tumor grade was specified, grade 1 and grade 2 tumors were most common (15\% and $16 \%$, respectively). The report from the Netherlands Cancer Registry also included a multivariate analysis of survival for patients with rare breast neoplasms, using invasive ductal carcinoma as a standard. In comparison to this invasive ductal carcinoma, patients with papillary carcinoma appeared to have prolonged survival, albeit with a wide confidence interval (odds ratio for survival 0.57; 95\% CI 0.2-1.6).

An analysis of the California Cancer Registry provides a more specific analysis of intracystic papillary carcinoma.[4] Between 1988 and 2005, a total of 917 cases were identified from this population-based registry. Of these, 427 were classified as intracystic papillary carcinoma in situ, whereas 490 cases were classified as invasive disease. The median age reported in this series was 69.5 years (range 27 to 99 years), with $3.5 \%$ of diagnoses occurring in men. Notably, the male incidence of intracystic papillary carcinoma appears to be substantially higher than the total reported incidence of male breast cancer (constituting approximately $1 \%$ of invasive tumors). Looking specifically at cases of invasive cancer, the majority of cases were localized at the time of diagnosis (89.6\%). Approximately $7.8 \%$ of cases were noted to have associated regional disease, with extension into adjacent tissue or axillary involvement, and only 2 cases $(0.4 \%)$ possessed distant metastases at the time of diagnosis.

Survival data from this registry analysis yielded interesting results. At 5 years, it appeared that there was a borderline statistical difference in overall cumulative survival favoring intracystic papillary carcinoma in situ as compared to invasive disease $(85.0 \%$ versus $75.0 \%$, $\mathrm{P}=0.05$ ) (Figure 10). However, at 10 years, no such difference was elicited between invasive and in situ disease (61.7\% versus $60.6 \%, \mathrm{P}=0.08)$. Comparison of cases to age-matched controls from the general population was also performed to specifically examine the effect of intracystic papillary carcinoma on survival. Again, at a 10 year interval, there were no differences in relative cumulative survival in patients with in situ and invasive disease ( $96.8 \%$ versus $94.4 \%, \mathrm{P}=0.75$ ). The lack of survival difference appears between in situ and invasive disease appears to be quite unique when juxtaposed against other tumor histologies; certainly, this may be a reflection of a relatively occult disease course.

\section{Papillary Carcinoma in Males}

Concordant with data reported from the California Cancer Registry, a number of series indicate a greater incidence of papillary carcinoma amongst males. With respect to in situ disease, a Surveillance, Epidemiology, and End Results (SEER) database review assessed 280 and 53,928 cases of in situ breast cancer in males and females, respectively, diagnosed between 1973 and 2001.[32] Amongst various histologic subtypes assessed, only papillary in situ breast cancer and invasive architectural types were more common in men. A separate review of 759 archived cases of invasive male breast cancer included 34 cases of papillary carcinoma.[33] Comparison of this proportion to the published frequency in women suggested a two-fold increase in the incidence of invasive papillary carcinoma in males. In fact, invasive papillary carcinoma constituted the most frequent rare histology encountered in males. The aforementioned series are bolstered by numerous case reports in the available literature. A MEDLINE database search using the terms male and papillary carcinoma yielded ten cases reports of papillary carcinomas of the male breast over the past decade alone (Table 1).[34-42] 


\section{TREATMENT}

The Netherlands Cancer Registry offers a perspective on different therapeutic modalities rendered in practice for patients with papillary carcinoma.[2] Again, this dataset comes with the caveat of including multiple histologic subtypes, as denoted in the previous section. In reporting treatment strategy, patients were stratified by age (either less than 70 , or 70 or greater). Amongst patients less than 70, with treatment data available for 58 patients, 13 patients $(31 \%)$ received surgery alone, while 26 patients $(45 \%)$ received surgery with radiation therapy. Systemic therapy was administered with surgery in 6 patients $(10 \%)$, and tri-modality therapy (surgery, radiation and chemotherapy) was rendered in an additional 6 patients (10\%). Treatment data was available for 48 patients aged 70 or greater, with 14 patients (29\%) receiving surgery alone and 20 patients (42\%) receiving surgery and radiation therapy. As compared to the younger cohort, a similar proportion of patients received surgery with systemic therapy and tri-modality therapy (13\% in both categories).

Outside of this large registry analysis including multiple rare tumor types, there are limited studies focused solely on treatment strategies for papillary carcinoma. Carmen et al reported a series of 40 patients with one of three subtypes of papillary carcinoma: (1) papillary in situ disease (14 patients), (2) papillary in situ disease with associated ductal carcinoma in situ (DCIS; 13 patients), and (3) invasive papillary carcinoma with or without DCIS (13 patients).[43] Interestingly, the incidence of disease recurrence and death attributable to disease did not differ amongst any of the three groups. Furthermore, outcomes did not differ according to type of surgery (mastectomy or segmental mastectomy) or administration of radiation therapy.

A similarly sized experience reported by Fayanju et al identified 45 females with papillary carcinoma.[44] Using a similar classification scheme as the previous study, 21 patients were identified with papillary in situ disease, 18 with papillary in situ disease associated with DCIS, and 6 with invasive papillary carcinoma. In this study, patients with invasive features were more likely to undergo an axillary staging procedure (100\%) as compared to patients with in situ disease alone (29\%) or in situ disease and associated DCIS (28\%) $(\mathrm{P}<0.001)$. Furthermore, patients with in situ disease alone were more likely to receive radiation therapy as compared to patients in the remaining categories $(\mathrm{P}<0.001)$. Finally, endocrine therapy was more common in patients with invasive papillary carcinoma and in situ papillary carcinoma associated with DCIS. The rather discordant therapy of these three groups in the absence of definitive guidelines for management underscores the need for further treatment and outcome-related studies related to papillary carcinoma.

\section{CONCLUSIONS}

Emerging studies have defined specific histologic, molecular and radiographic features of both papillary carcinoma of the breast. Additionally, large series have defined prognosis of papillary carcinoma relative to other histologic subtypes. Future assessment should focus on treatment- and outcome-related analyses of papillary carcinoma, given the divergent array of approaches currently utilized (as outlined in limited available series). These analyses should stratify for the various subtypes of papillary carcinoma, recognizing the heterogeneity of this classification. Furthermore, novel gene panels may serve as a potential decision tool in this rare entity. Assessment of 25,475 tumors by a validated 21-gene assay included over 100 invasive papillary carcinomas (0.2\%).[45] A median recurrence score of 7.8 was determined for this population, indicating a relatively low likelihood of recurrence. Prospective use of this gene panel is currently underway for adenocarcinomas of the breast; it will be interesting to see whether the use of this or other evolving molecular assays are applicable in the context of rare histologies such as papillary carcinomas.[46] 


\section{Acknowledgments}

Acknowledgment of Research Support: Dr. Pals's efforts are supported by CBCRP 15IB-0140 (California Breast Cancer Research Program Junior IDEA Award) and NIH K12 2K12CA001727-16A1.

\section{References}

1. Jemal A, Siegel R, Ward E, Hao Y, Xu J, Thun MJ. Cancer Statistics, 2009. CA A Cancer Journal for Clinicians. 2009; 59(4):225-49. [PubMed: 19474385]

2. Louwman MWJ, Vriezen M, Beek MWPMv, Nolthenius-Puylaert MCBJET, Sangen MJCvd, Roumen RM, et al. Uncommon breast tumors in perspective: Incidence, treatment and survival in the Netherlands. International Journal of Cancer. 2007; 121(1):127-35.

3. McCulloch GL, Evans AJ, Yeoman L. Radiological features of papillary carcinoma of the breast. Clin Radiol. 1997; 73:865-8. [PubMed: 9392466]

4. Grabowski J, Salzstein SL, Sadler GR, Blair S. Intracystic papillary carcinoma. Cancer. 2008; 113(5):916-20. [PubMed: 18661510]

5. Mulligan AM, O’Malley FP. Papillary lesions of the breast: a review. Adv Anat Pathol. 2007 Mar; 14(2):108-19. [PubMed: 17471117]

6. Collins LC, Schnitt SJ. Papillary lesions of the breast: selected diagnostic and management issues. Histopathology. 2008 Jan; 52(1):20-9. [PubMed: 18171414]

7. Ueng SH, Mezzetti T, Tavassoli FA. Papillary neoplasms of the breast: a review. Arch Pathol Lab Med. 2009 Jun; 133(6):893-907. [PubMed: 19492881]

8. Page DL, Salhany KE, Jensen RA, Dupont WD. Subsequent breast carcinoma risk after biopsy with atypia in a breast papilloma. Cancer. 1996 Jul 15; 78(2):258-66. [PubMed: 8674001]

9. Hill CB, Yeh IT. Myoepithelial Cell Staining Patterns of Papillary Breast Lesions. American Journal of Clinical Pathology. 2005; 123(1):36-44. [PubMed: 15762278]

10. Collins LC, Carlo VP, Hwang H, Barry TS, Gown AM, Schnitt SJ. Intracystic papillary carcinomas of the breast: a reevaluation using a panel of myoepithelial cell markers. Am J Surg Pathol. 2006 Aug; 30(8):1002-7. [PubMed: 16861972]

11. Esposito NN, Dabbs DJ, Bhargava R. Are encapsulated papillary carcinomas of the breast in situ or invasive? A basement membrane study of 27 cases. Am J Clin Pathol. 2009 Feb; 131(2):228-42. [PubMed: 19141383]

12. Maluf HM, Koerner FC. Solid papillary carcinoma of the breast. A form of intraductal carcinoma with endocrine differentiation frequently associated with mucinous carcinoma. Am J Surg Pathol. 1995 Nov; 19(11):1237-44. [PubMed: 7573685]

13. Nassar H, Qureshi H, Volkanadsay N, Visscher D. Clinicopathologic analysis of solid papillary carcinoma of the breast and associated invasive carcinomas. Am J Surg Pathol. 2006 Apr; 30(4): 501-7. [PubMed: 16625097]

14. Moritani S, Ichihara S, Kushima R, Okabe H, Bamba M, Kobayashi T, et al. Myoepithelial cells in solid variant of intraductal papillary carcinoma of the breast: a potential diagnostic pitfall and a proposal of an immunohistochemical panel in the differential diagnosis with intraductal papilloma with usual ductal hyperplasia. Virchows Archiv. 2007; 450(5):539-47. [PubMed: 17377808]

15. Nicolas MM, Wu Y, Middleton LP, Gilcrease MZ. Loss of myoepithelium is variable in solid papillary carcinoma of the breast. Histopathology. 2007; 51(5):657-65. [PubMed: 17927587]

16. Fisher ER, Palekar AS, Redmond C. Pathologic findings from the National Surgical Adjuvant Breast Project (protocol no.4) VI. Invasive papillary cancer. Am J Clin Pathol. 1980; 73:313-22. [PubMed: 7361710]

17. Walsh MM, Bleiweiss IJ. Invasive micropapillary carcinoma of the breast: Eighty cases of an underrecognized entity. Human pathology. 2001; 32(6):583-9. [PubMed: 11431712]

18. Nassar H, Wallis T, Andea A, Dey J, Adsay V, Visscher D. Clinicopathologic Analysis of Invasive Micropapillary Differentiation in Breast Carcinoma. Mod Pathol. 14(9):836-41. [PubMed: 11557778] 
19. Pettinato G, Manivel CJ, Panico L, Sparano L, Petrella G. Invasive Micropapillary Carcinoma of the Breast. American Journal of Clinical Pathology. 2004 June; 121(6):857-66. [PubMed: 15198358]

20. Shah VI, Flowers CI, Douglas-Jones AG, Dallimore NS, Rashid M. Immunohistochemistry increases the accuracy of diagnosis of benign papillary lesions in breast core needle biopsy specimens. Histopathology. 2006 May; 48(6):683-91. [PubMed: 16681684]

21. Grin A, O’Malley FP, Mulligan AM. Cytokeratin 5 and estrogen receptor immunohistochemistry as a useful adjunct in identifying atypical papillary lesions on breast needle core biopsy. Am J Surg Pathol. 2009 Nov; 33(11):1615-23. [PubMed: 19675450]

22. Rabban JT, Koerner FC, Lerwill MF. Solid papillary ductal carcinoma in situ versus usual ductal hyperplasia in the breast: a potentially difficult distinction resolved by cytokeratin $5 / 6$. Hum Pathol. 2006 Jul; 37(7):787-93. [PubMed: 16784976]

23. Tan PH, Aw MY, Yip G, Bay BH, Sii LH, Murugaya S, et al. Cytokeratins in papillary lesions of the breast: is there a role in distinguishing intraductal papilloma from papillary ductal carcinoma in situ? Am J Surg Pathol. 2005 May; 29(5):625-32. [PubMed: 15832086]

24. Grin A, O’Malley FP, Mulligan AM. Cytokeratin 5 and Estrogen Receptor Immunohistochemistry as a Useful Adjunct in Identifying Atypical Papillary Lesions on Breast Needle Core Biopsy. The American Journal of Surgical Pathology. 2009; 33(11):1615-23.10.097/PAS.0b013e3181aec446 [PubMed: 19675450]

25. Cristofano CD, Mrad K, Zavaglia K, Bertacca G, Aretini P, Cipollini G, et al. Papillary lesions of the breast: a molecular progression? Breast Cancer Research and Treatment. 2005; 90(1):71-6. [PubMed: 15770529]

26. Tsuda H, Takarabe T, Inazawa J, Hirohashi S. Detection of Numerical Alterations of Chromosomes 3, 7, 17 and X in Low-grade Intracystic Papillary Tumors of the Breast by Multicolor Fluorescence In Situ Hybridization. Breast Cancer. 1997 Dec; 4(4):247-52. [PubMed: 11091608]

27. Ganesan S, Karthik G, Joshi M, Damodaran V. Ultrasound spectrum in intraductal papillary neoplasms of breast. Br J Radiol. October 1; 2006 79(946):843-9. [PubMed: 16641415]

28. Kim TH, Kang DK, Kim SY, Lee EJ, Jung YS, Yim H. Sonographic Differentiation of Benign and Malignant Papillary Lesions of the Breast. J Ultrasound Med. January 1; 2008 27(1):75-82. [PubMed: 18096733]

29. Lam WWM, Chu WCW, Tang APY, Tse G, Ma TKF. Role of Radiologic Features in the Management of Papillary Lesions of the Breast. Am J Roentgenol. May 1; 2006 186(5):1322-7. [PubMed: 16632726]

30. Linda A, Londero V, Mazzarella F, Zuiani C, Bazzocchi M. Rare breast neoplasms: is there any peculiar feature on magnetic resonance mammography? La Radiologia Medica. 2007; 112(6):85062. [PubMed: 17885743]

31. Bernik SF, Troob S, Ying BL, Simpson SA, Axelrod DM, Siegel B, et al. Papillary lesions of the breast diagnosed by core needle biopsy: 71 cases with surgical follow-up. American journal of surgery. 2009; 197(4):473-8. [PubMed: 18723154]

32. Anderson W, SSD. In situ male breast carcinoma in the Surveillance, Epidemiology, and End Results database of the National Cancer Institute. Cancer. 2005 Oct 15; 104(8):1733-41. [PubMed: 16138363]

33. Burga A, Fadare O, Lininger R, Tavassoli F. Invasive carcinomas of the male breast: a morphologic study of the distribution of histologic subtypes and metastatic patterns in 778 cases. Virchows Archiv. 2006; 449(5):507-12. [PubMed: 17058095]

34. Anan H, Okazaki M, Fujimitsu R, Hamada Y, Sakata N, Nanbu M. Intracystic papillary carcinoma in the male breast. A case report. Acta Radiol. 2000; 41(3):227-9. [PubMed: 10866076]

35. Bhatia A, Saikia Uma Nahar, Kumar Y. Rare Coexistence of Invasive Papillary Carcinoma with Infiltrating Ductal Carcinoma in Male Breast: Report of a Case. International Journal of Surgical Pathology. July 1; 2008 16(3):311-3. [PubMed: 18573787]

36. Blaumeiser B, Tjalma W, Verslegers I, et al. Invasive papillary carcinoma of the male breast. European Radiology. 2002; 12(9):2207-10. [PubMed: 12195471] 
37. Dragoumis D, Tsiftsoglou A. Intracystic papillary carcinoma associated with ductal carcinoma in situ in a male breast. Journal of Postgraduate Medicine. 2008; 54(1):39-40. [PubMed: 18296806]

38. Imoto S, Hasebe T. Intracystic papillary carcinoma of the breast in male: case report and review of the Japanese literature. Jpn J Clin Oncol. 1998; 28(8):517-20. [PubMed: 9769788]

39. Pacelli A, Bock BJ, Jensen EA, Heerden JA, Reynolds C. Intracystic papillary carcinoma of the breast in a male patient diagnosed by ultrasound-guided core biopsy: a case report. Breast J. 2002 Nov-Dec; 8(6):387-90. [PubMed: 12390365]

40. Takayuki K, Takashi F, Eriko I, Miyuki T, Sadako A-T, Tadashi H. Intracystic papillary carcinoma of the breast in a male patient diagnosed by core needle biopsy: a case report. Breast (Edinburgh, Scotland). 2005; 14(4):322-4.

41. Tochika N, Takano A, Yoshimoto T, Tanaka J, Sugimoto T, Kobayashi M, et al. Intracystic carcinoma of the male breast: report of a case. Surg Today. 2001; 31(9):806-9. [PubMed: 11686560]

42. Zeppa P, Mascolo M, Zabatta A, Finelli L, Vetrani A, Palombini L. Aggressive papillary male breast carcinoma on fine-needle cytology sample. Diagn Cytopathol. 2003; 29(6):360-3. [PubMed: 14648798]

43. Solorzano CC, Middleton LP, Hunt KK, Mirza N, Meric F, Kuerer HM, et al. Treatment and outcome of patients with intracystic papillary carcinoma of the breast. American journal of surgery. 2002; 184(4):364-8. [PubMed: 12383904]

44. Fayanju OM, Ritter J, Gillanders WE, Eberlein TJ, Dietz JR, Aft R, et al. Therapeutic management of intracystic papillary carcinoma of the breast: the roles of radiation and endocrine therapy. American journal of surgery. 2007; 194(4):497-500. [PubMed: 17826064]

45. Baehner FL, Watson D, Ballard JT, Palmer G, Shak S. Gene expression by standardized quantitative RT-PCR in the special histologic subtypes of estrogen receptor positive invasive breast cancer. Breast Cancer Res Treat. 2008 [Abstr 3028].

46. Welsch C, Brown C, Goodrich-Smith M, Van J, Denenberg B, Anderson T, et al. Inhibition of mammary tumorigenesis in carcinogen-treated Lewis rats by suppression of prolactin secretion. $\mathrm{J}$ Natl Cancer Inst. 1979; 63:1121 - 4. [PubMed: 91695] 


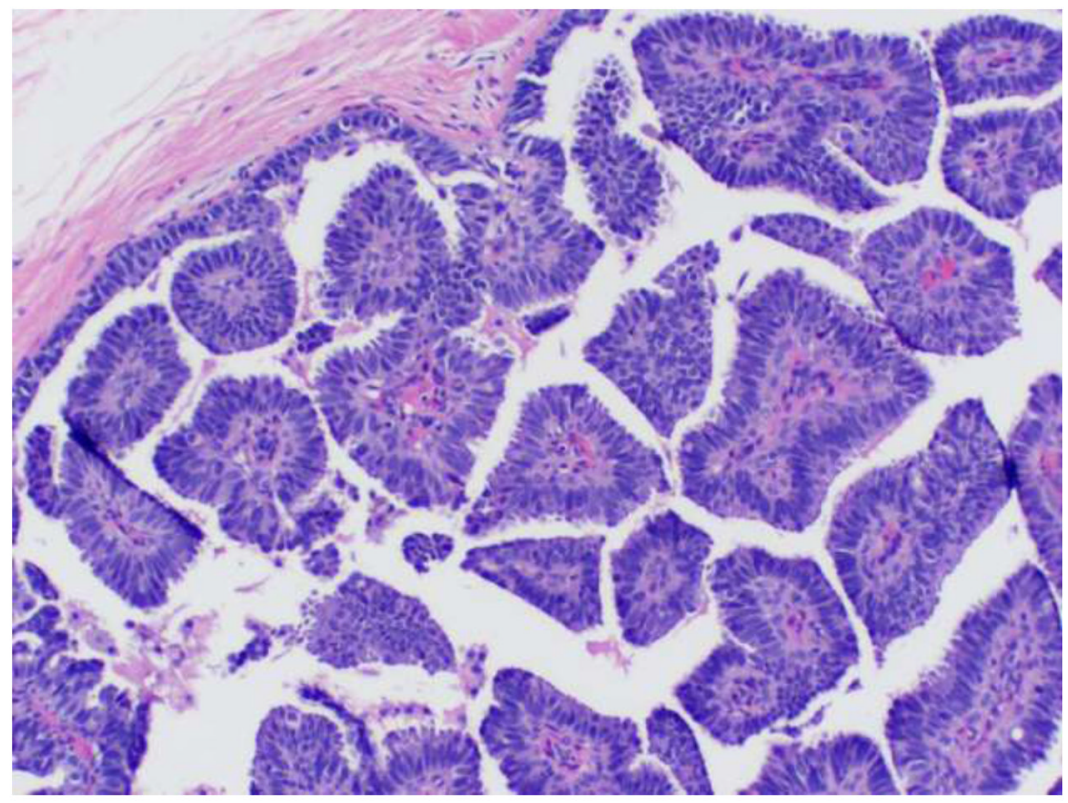

Figure 1.

Papillary DCIS. 


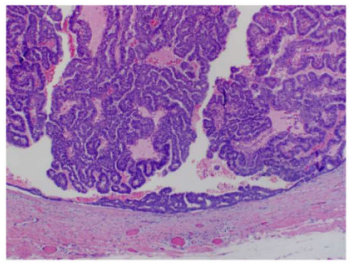

Figure 2.

Encapsulated (intracystic) papillary carcinoma. 


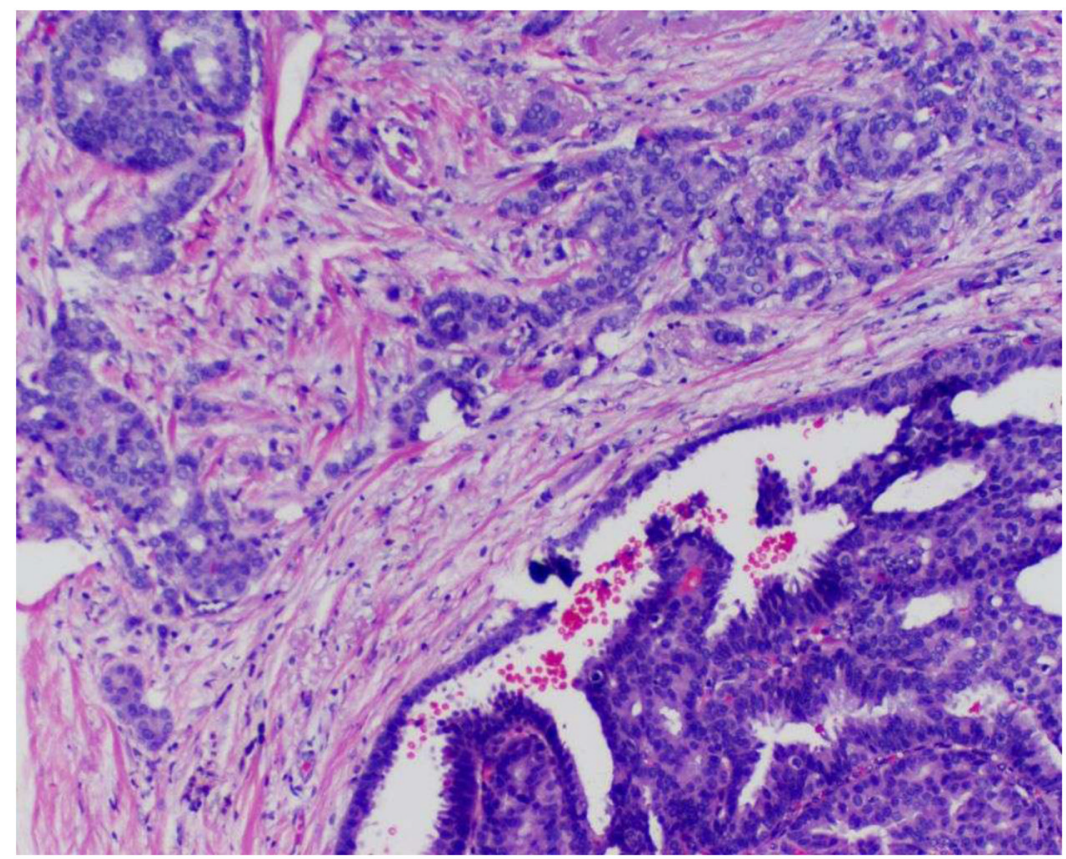

Figure 3.

Encapsulated papillary carcinoma with an invasive component. 


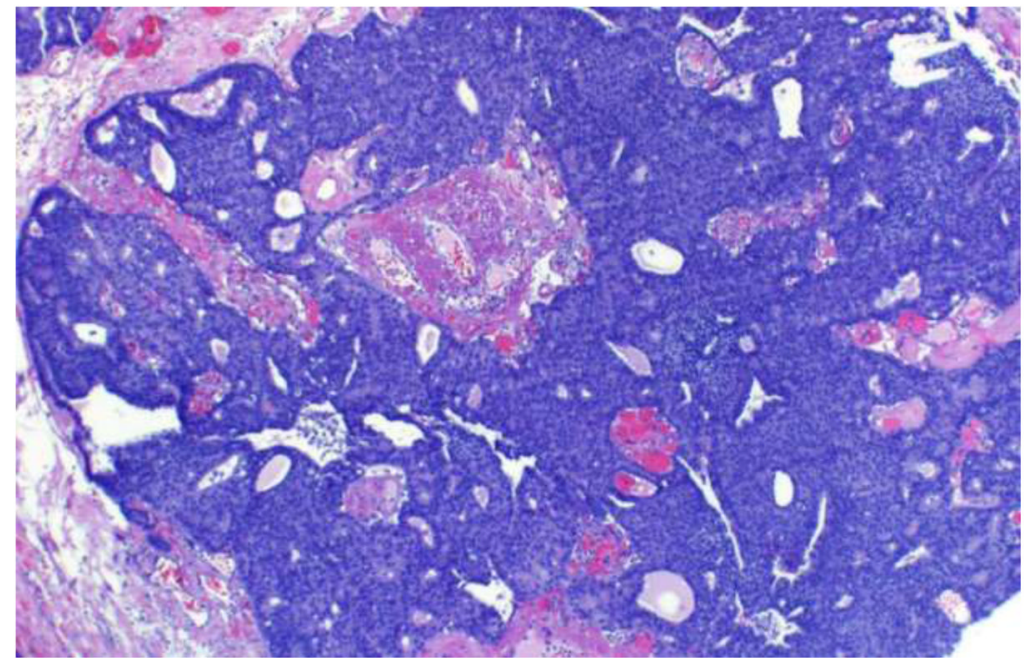

Figure 4.

Solid papillary carcinoma, low magnification. 


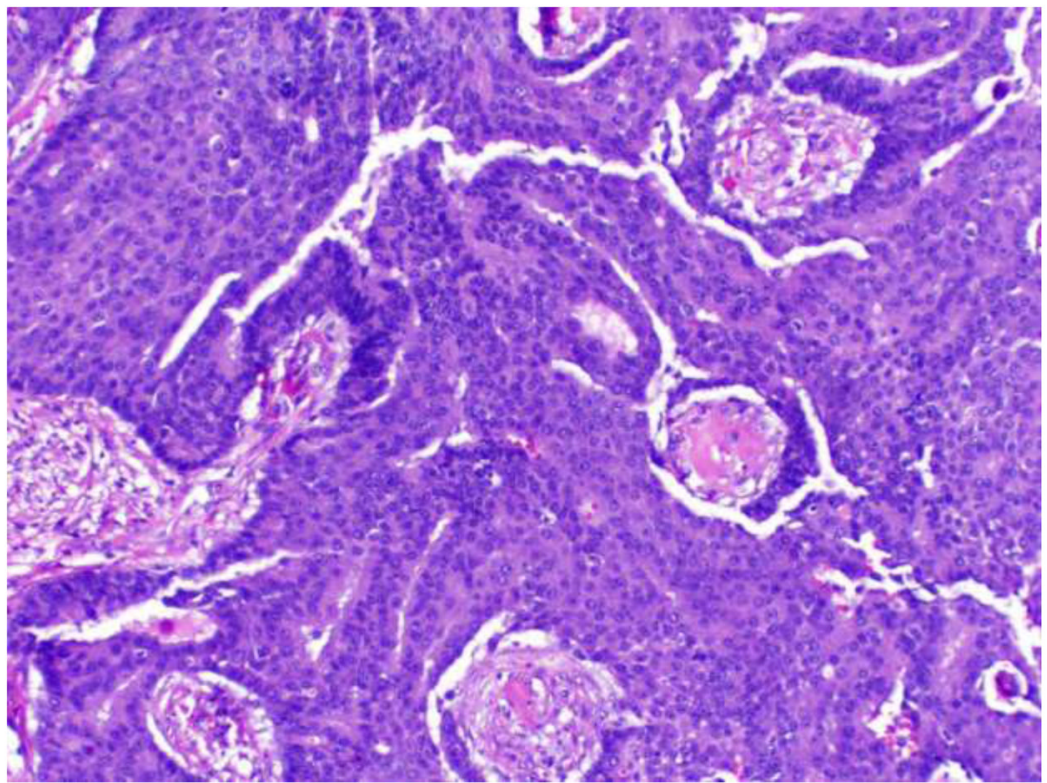

Figure 5.

Solid papillary carcinoma, intermediate magnification. 


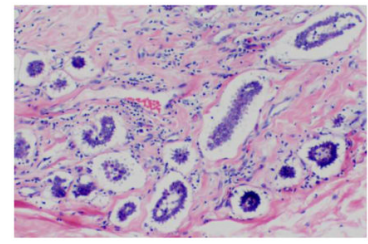

Figure 6.

Invasive micropapillary carcinoma. 


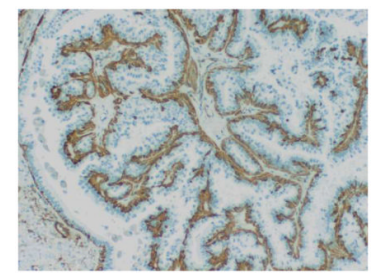

Figure 7.

Intraductal papilloma. Immunohistochemistry for calponin decorates myoepithelial cells in the intraluminal papillary fronds and surrounding the involved duct. 


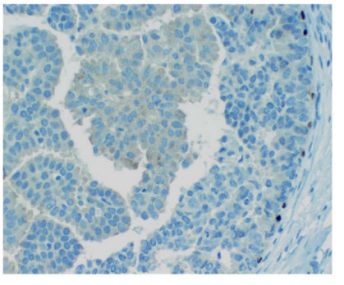

Figure 8.

Papillary DCIS. P63 immunohistochemistry shows discontinuous staining along the periphery of the duct. No myoepithelial cells are detected within the papillae. 

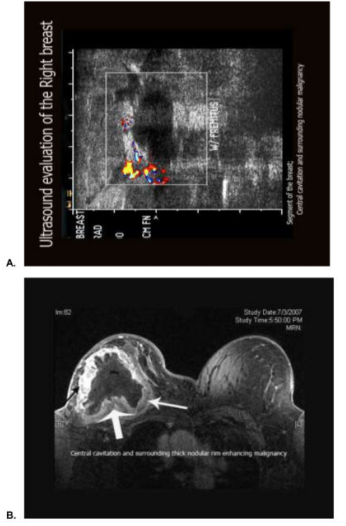

Figure 9.

Ultrasonographic (A) and magnetic resonance mammographic (B) characterization of a large, invasive papillary carcinoma diagnosed. (Courtesy of Lalit Vora, M.D., City of Hope Comprehensive Cancer Center, Duarte, CA.) 


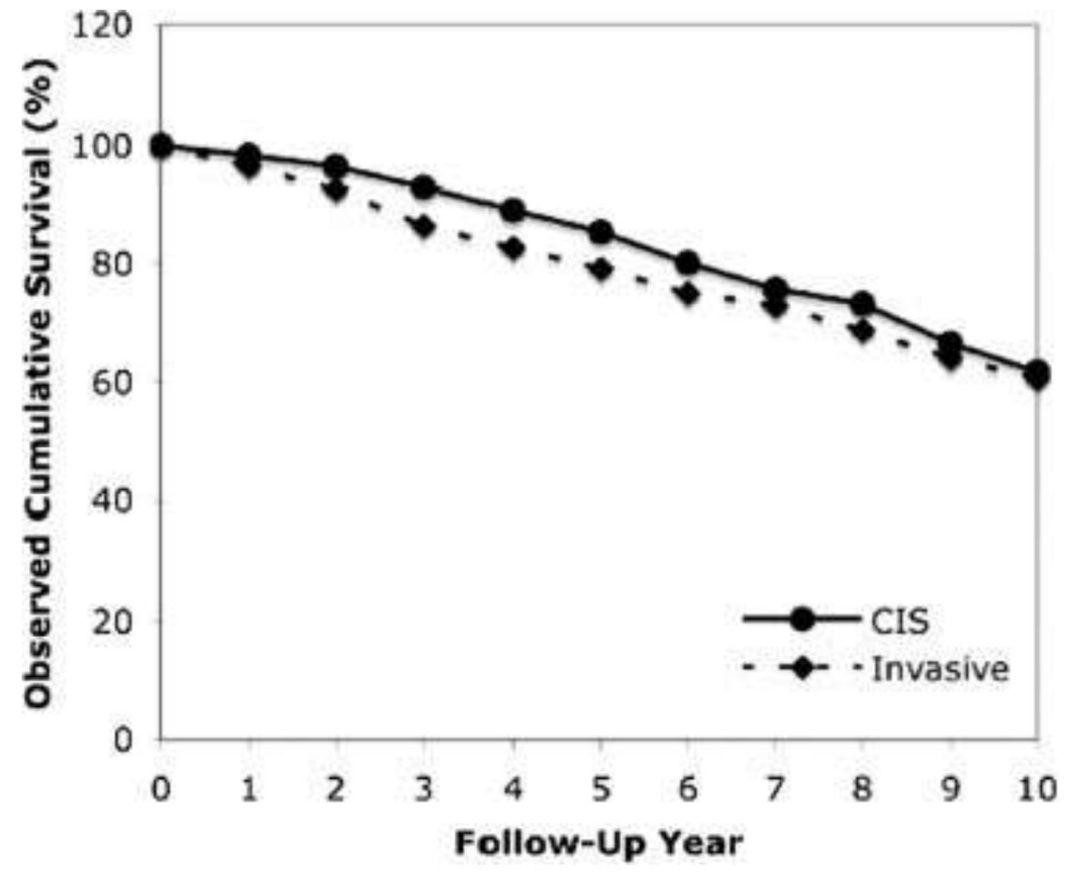

Figure 10.

Observed cumulative survival in patients with invasive and in situ papillary carcinoma of the breast. (Reproduced with permission from Grabowski J, Salzstein SL, Sadler GR, et al: Intracystic papillary carcinoma. Cancer 113:916-920, 2008.) 
Table 1

Case reports of papillary carcinoma of the breast in males identified from a MEDLINE database search using terms male and papillary carcinoma.

\begin{tabular}{|c|c|c|}
\hline Author & Year & Description of Case \\
\hline Anan et al[34] & 2000 & 75 year old Japanese male with papillary carcinoma diagnosed by pneumocystography and ultrasonography. \\
\hline Bhatia et al [35] & 2008 & $\begin{array}{l}35 \text { year old male with invasive papillary carcinoma with concomitant infiltrating ductal carcinoma in a } \\
\text { separate lesions. }\end{array}$ \\
\hline Blaumeiser et al[36] & 2002 & 77 year old male with invasive papillary carcinoma diagnosed by magnetic resonance mammography. \\
\hline Dragoumis et al[37] & 2008 & 75 year old male with invasive papillary carcinoma with concomitant ductal carcinoma in situ. \\
\hline Imoto et al[38] & 1998 & $\begin{array}{l}62 \text { year old Japanese male with invasive papillary carcinoma diagnosed upon aspiration of a palpated breast } \\
\text { mass. }\end{array}$ \\
\hline Pacelli et al[39] & 2007 & 67 year old male with papillary carcinoma diagnosed after ultrasound- guided core biopsy. \\
\hline Takayuki et al[40] & 2005 & 71 year old male with papillary carcinoma diagnosed after core needle biopsy. \\
\hline Tochika et al[41] & 2001 & $\begin{array}{l}66 \text { year old male with papillary carcinoma diagnosed using dynamic magnetic resonance imaging and power } \\
\text { Doppler ultrasonography. }\end{array}$ \\
\hline Zeppa et al[42] & 2003 & 55 year old male with aggressive papillary carcinoma with invasion of the thoracic wall. \\
\hline
\end{tabular}

